\title{
Capacitation and acrosome reaction differences of bovine, mouse and porcine spermatozoa in responsiveness to estrogenic compounds
}

\author{
Do-Yeal Ryu', Ye-Ji Kim, June-Sub Lee, Md Saidur Rahman, Woo-Sung Kwon, Sung-Jae Yoon
} and Myung-Geol Pang*

\begin{abstract}
Background: Endocrine disruptors are exogenous substance, interfere with the endocrine system, and disrupt hormonal functions. However, the effect of endocrine disruptors in different species has not yet been elucidated. Therefore, we investigated the possible effects of 17ß-estradiol (E2), progesterone (P4), genistein (GEN) and 4-tert-octylphenol (OP), on capacitation and the acrosome reaction in bovine, mouse, and porcine spermatozoa. In this in vitro trial, spermatozoa were incubated with $0.001-100 \mu \mathrm{M}$ of each chemical either 15 or $30 \mathrm{~min}$ and then assessed capacitation status using chlortetracycline staining.

Results: E2 significantly increased capacitation and the acrosome reaction after 30 min, while the acrosome reaction after 15 min incubation in mouse spermatozoa. Simultaneously, capacitation and the acrosome reaction were induced after 15 and 30 min incubation in porcine spermatozoa, respectively. Capacitation was increased in porcine spermatozoa after 15 min incubation at the lowest concentration, while the acrosome reaction was increased in mouse spermatozoa after $30 \mathrm{~min}(P<0.05)$. E2 significantly increased the acrosome reaction in porcine spermatozoa, but only at the highest concentration examined $(P<0.05)$. P4 significantly increased the acrosome reaction in bovine and mouse spermatozoa treated for $15 \mathrm{~min}(P<0.05)$. The same treatment significantly increased capacitation in porcine spermatozoa $(P<0.05)$. P4 significantly increased capacitation in mouse spermatozoa treated for $30 \mathrm{~min}(P<0.05)$. GEN significantly increased the acrosome reaction in porcine spermatozoa treated for 15 and $30 \mathrm{~min}$ and in mouse spermatozoa treated for $30 \mathrm{~min}$ $(P<0.05)$. OP significantly increased the acrosome reaction in mouse spermatozoa after 15 min $(P<0.05)$. Besides, when spermatozoa were incubated for $30 \mathrm{~min}$, capacitation and the acrosome reaction were higher than 15 min incubation in E2 or GEN. Furthermore, the responsiveness of bovine, mouse and porcine spermatozoa to each chemical differed.

Conclusions: In conclusion, all chemicals studied effectively increased capacitation and the acrosome reaction in bovine, mouse, and porcine spermatozoa. Also we found that both E2 and P4 were more potent than environmental estrogens in altering sperm function. Porcine and mouse spermatozoa were more responsive than bovine spermatozoa.
\end{abstract}

Keywords: Capacitation, Acrosome reaction, Estrogen, Endocrine disruptor, Spermatozoa

\footnotetext{
* Correspondence: mgpang@cau.ac.kr

${ }^{\dagger}$ Equal contributors

Department of Animal Science and Technology, Chung-Ang University, 4726

Seodong-daero, Anseong 456-756, Gyeonggi-Do, Republic of Korea
} 


\section{Background}

Estrogens play key roles in regulating various physiological phenomena related to normal growth, development, and reproduction in mammals [1]. Although estrogens have been considered to be female reproductive hormones, recent evidence has indicated that they play an important role in the development and regulation of the male reproductive system [2]. Estrogens were detected in male serum as well as in the male gonad [3].

Recent reports have shown that endocrine disruptors are exogenous substances that interfere with the endocrine system and might disrupt hormone function in various wildlife species. Moreover, it has also been suggested that these compounds may be responsible for a variety of reproductive disturbances in men, including possible declines in sperm concentration $[4,5]$. Many non-steroid compounds in the environment have been found to exhibit estrogenic activity [6,7], where these include natural phytoestrogens, pesticides, and industrial products with various homologies to estradiol that can bind with estrogen receptors, acting either as agonists or antagonists [8]. Most studies of xenobiotics have focused on long-term developmental effects on the testis, the male reproductive tract and semen quality. When xenobiotics are used at relatively high dosages, they disrupt spermatogenesis and hence decrease overall male fertility [9].

Luconi et al. [2] have identified and characterized novel nongenomic estrogen receptors on the cell membrane of human spermatozoa that interfere with the effects of progesterone (P4). Thus, spermatozoa may represent a suitable model to study the possible effects of estrogenic xenobiotics on the function of spermatozoa. Adeoya-Osiguwa et al. [10] recently reported evidence that very low dosages of several xenobiotics have direct effects on the function of spermatozoa, significantly accelerating capacitation and the acrosome reaction in mice. However, there are no data on these effects in domestic animals. Examination of the physiological and nonphysiological effects of estrogenic xenobiotics on sperm function requires a high-quality in vitro test system [11].

To look for any significant effects of treatment length and concentration on the rate of capacitation and acrosome reaction, we examined the effects of $17 \beta$-estradiol (E2), P4, and two estrogenic compounds, namely genistein (GEN) and 4-tert-octylphenol (OP) on bovine, mouse and porcine spermatozoa.

\section{Methods}

All procedures were performed according to guidelines for the ethical treatment of animals and approved by Institutional Animal Care and Use Committee of ChungAng University (Approval no. 12-0021).

\section{Medium and reagents}

Throughout this study, bovine, mouse and porcine spermatozoa were treated in modified TCM 199 that comprised TCM 199 with Earle's salts containing 10\% heat-inactivated fetal calf serum $(\mathrm{v} / \mathrm{v}), 0.91 \mathrm{mM}$ sodium pyruvate, $3.05 \mathrm{mM}$ D-glucose, $2.92 \mathrm{mM}$ calcium lactate, $50 \mathrm{IU} / \mathrm{l}$ penicillin $\mathrm{G}$, and $30 \mu \mathrm{g} / \mathrm{ml}$ streptomycin sulfate. A stock solution of $1000 \mu \mathrm{M}$ E2 and P4 (Sigma-Aldrich, St Louis, MO, USA) was prepared in dimethyl sulfoxide (DMSO) and stored at $-20^{\circ} \mathrm{C}$. Working stock solutions were prepared daily by first diluting the initial stock solution in 10\% DMSO: 0.9\% $\mathrm{NaCl}(1: 1)$. This solution was used for subsequent dilutions of the standard medium. The other stock solutions $(100 \mu \mathrm{M})$ of GEN (Sigma-Aldrich, St Louis, MO, USA), P4 and OP (Sigma-Aldrich, St Louis, MO, USA) were prepared in absolute ethanol and stored at $-20^{\circ} \mathrm{C}$. The working stock solutions were prepared daily using standard medium as diluent.

\section{Preparation of spermatozoa}

Frozen bovine semen and liquid porcine semen were obtained from the National Agriculture Cooperation Federation (Goyang, Gyeonggi, Korea) and Yonam Genetics, Inc. (Chonan, Chungnam, Korea), respectively. Epididymal mouse sperm cells were collected from 9-week-old male ICR mice (Central Lab. Animal Inc, Seoul, Korea). For frozen-thawed bovine and liquid porcine semen, sperm cells were centrifuged at $500 \times \mathrm{g}$ for $3 \mathrm{~min}$ and the sperm pellets were diluted with modified TCM 199 with or without chemicals. Subsequently, suspensions were centrifuged at $500 \times \mathrm{g}$ for $3 \mathrm{~min}$ and sperm pellets were diluted with modified TCM 199 solution containing one of the chemicals examined. These were incubated for $15 \mathrm{~min}$ or $30 \mathrm{~min}$ in an atmosphere of $5 \% \mathrm{CO}_{2}$ at $39^{\circ} \mathrm{C}$. For mouse spermatozoa, caudal epididymal spermatozoa from three mature ICR males were released into sterile plastic dishes containing modified TCM 199 (0.1\% BSA). Suspensions were then allowed to disperse for $5 \mathrm{~min}$ on a warming tray and motile sperm cells were collected. Sperm cells were diluted with modified TCM 199 containing one of the chemicals examined. These samples were incubated for $15 \mathrm{~min}$ or $30 \mathrm{~min}$ in an atmosphere of 5\% $\mathrm{CO}_{2}$ at $37^{\circ} \mathrm{C}$. The analysis of each condition for each animal was replicated 3 times.

\section{Combined Hoechst 33258/chlortetracycline fluorescence assessment of spermatozoa (H33258/CTC)}

The dual staining method performed was based on that described by Perez et al. [12], with some modifications. Briefly, $135 \mu \mathrm{l}$ of semen $\left(2 \times 10^{8} \mathrm{cell} / \mathrm{ml}\right)$ was added to

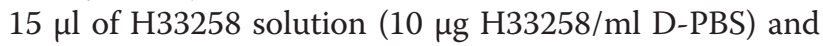
incubated at $37^{\circ} \mathrm{C}$ for $10 \mathrm{~min}$ in a light-shielded water bath. Excess dye was removed by layering the mixture over $250 \mu \mathrm{l}$ of $2 \%$ (w/v) polyvinylpyrrolidone (PVP) in PBS that had been centrifuged at $400 \times \mathrm{g}$ for $10 \mathrm{~min}$. The supernatant was discarded and the pellet was resuspended 
in $700 \mu \mathrm{l}$ of PBS and $500 \mu \mathrm{l}$ of this solution was added to $500 \mu \mathrm{l}$ of a freshly prepared CTC solution $(1.3 \mathrm{mg}$ CTC in $5 \mathrm{ml}$ buffer: $20 \mu \mathrm{M}$ Tris, $130 \mu \mathrm{M} \mathrm{NaCl}, 5 \mu \mathrm{M}$ cystein). After $20 \mathrm{sec}$, the reaction was stopped by the addition of $10 \mu \mathrm{l}$ of $12.5 \%(\mathrm{v} / \mathrm{v})$ glutaraldehyde solution in $1 \mathrm{M}$ Tris buffer and maintained at $4^{\circ} \mathrm{C}$ in the dark until evaluation (within $24 \mathrm{~h}$ of preparation). Samples were observed with a Nikon microphot-FXA under epifluorescence illumination using UV BP 340-380/LP 425 and BP 450-490/LP 515 excitation/emission filters for H33258 and CTC, respectively. Spermatozoa were classified as shown in Table 1: dead (D type, when nuclei showed bright blue fluorescence over the sperm head), live noncapacitated ( $\mathrm{F}$ type, bright green fluorescence distributed uniformly over the entire sperm head, with or without stronger fluorescent line at the equatorial segment), live capacitated (B type, green fluorescence over acrosomal region and dark postacrosome), or live acrosome-reacted (AR type, spermatozoa showing a mottled green fluorescence over head, green fluorescence only in post acrosomal region or no fluorescence on the head) [13]. All spermatozoa had bright green fluorescent midpieces. Two slides per sample were evaluated, with at least 100 spermatozoa per slide.

\section{Statistics}

The data were analyzed using ANOVA performed with SPSS software (v. 12.0; Chicago, Illinois, USA). This test compares responses within replicates for a significant difference to be obtained, a consistent and reasonable magnitude is required between control and treated samples. A value of $P<0.05$ was considered statistically significant.

\section{Results}

This study investigated the possible effects of E2, P4, and two estrogenic environmental estrogens, GEN, and OP, on capacitation and acrosome reaction in bovine, mouse, and porcine spermatozoa in vitro. Bovine and porcine spermatozoa suspensions were incubated with 0.001-100 $\mu \mathrm{M}$ E2, P4, GEN, and OP for either 15 or $30 \mathrm{~min}$ at $39^{\circ} \mathrm{C}$ and then assessed using CTC fluorescence. Mouse spermatozoa were incubated at $37^{\circ} \mathrm{C}$.

Table 1 Determination of capacitation status patterns

\begin{tabular}{cc}
\hline Patterns (\%) & Description \\
\hline F & $\begin{array}{c}\text { Full fluorescence characteristic of ejaculated } \\
\text { spermatozoa: characteristic of uncapacitated } \\
\text { spermatozoa }\end{array}$ \\
B & $\begin{array}{c}\text { Banded, indicative of capacitated spermatozoa } \\
\text { and fluorescence only in the post acrosomal } \\
\text { region: characteristic of capacitated spermatozoa }\end{array}$ \\
AR & $\begin{array}{c}\text { Spermatozoa showing a mottled green fluorescence } \\
\text { over head, green fluorescence only in post acrosomal } \\
\text { region or no fluorescence on the head: typical } \\
\text { acrosome-reacted spermatozoa }\end{array}$ \\
\hline
\end{tabular}

Effects of E2 on bovine, mouse, and porcine spermatozoa A concentration-dependent pattern of capacitation was observed in mouse spermatozoa. E2 (0.001-100 $\mu \mathrm{M})$ significantly increased the acrosome reaction in mouse spermatozoa and capacitation in porcine spermatozoa after $15 \mathrm{~min}$ incubation $(P<0.05$, Figure $1 \mathrm{~B}$ and $\mathrm{C})$. Following $30 \mathrm{~min}$ exposure, E2 significantly increased both capacitation and the acrosome reaction in mouse spermatozoa, and significantly increased the acrosome reaction in porcine spermatozoa $(P<0.05)$ (Figure 2B and $C)$. No detectable effects were observed in bovine spermatozoa treated for $15 \mathrm{~min}$ or $30 \mathrm{~min}$ (Figures $1 \mathrm{~A}$ and $2 \mathrm{~A}$ ). The acrosome reaction in mouse spermatozoa was responded to E2 at higher concentrations $(10-100 \mu \mathrm{M})$ after $15 \mathrm{~min}$ incubation and responded to $0.001 \mu \mathrm{M}$ after 30 min incubation. Capacitation in mouse spermatozoa was increased significantly at higher concentrations $(10-100 \mu \mathrm{M})$ after $30 \mathrm{~min}$ incubation $(P<0.05)$ (Figures 1B and $2 \mathrm{~B})$. Capacitation was increased in porcine spermatozoa after 15 min incubation in E2 at a concentration of $0.001 \mu \mathrm{M}$. This effect decreased gradually with increasing doses (Figure 1C). E2 significantly increased the acrosome reaction in porcine spermatozoa, but only after $30 \mathrm{~min}$ incubation at the highest concentration $(P<0.05)$ (Figures $1 \mathrm{C}$ and $2 \mathrm{C}$ ).

Effects of $\mathrm{P} 4$ on bovine, mouse, and porcine spermatozoa After a 15-minute exposure to P4, a concentrationdependent effect on acrosome reaction was observed. P4 (0.001-100 $\mu \mathrm{M})$ significantly increased the acrosome reaction in both bovine and mouse spermatozoa treated for $15 \mathrm{~min}(P<0.05)$. This condition also significantly increased capacitation $(P<0.05)$ (Figure 3A and $\mathrm{B})$. No detectable acrosome reaction was observed in porcine spermatozoa (Figure 3C). P4 significantly increased capacitation in a dose-dependent manner in mouse spermatozoa treated for $30 \mathrm{~min}(P<0.05)$ (Figure 4B), while no detectable effects were observed in bovine or porcine spermatozoa (Figure 4A and C).

In bovine spermatozoa, the acrosome reaction was significantly increased after $15 \mathrm{~min}$ incubation at higher concentrations $(P<0.05)$ (Figure 3A) $(10-100 \mu \mathrm{M})$. The mouse spermatozoa first exhibited a response at $0.001 \mu \mathrm{M}$ (Figure 3B). However, no significant effects on acrosome reaction were observed after $30 \mathrm{~min}$ incubation in any treated spermatozoa (Figure 4).

\section{Effects of GEN on bovine, mouse, and porcine spermatozoa}

GEN (0.001-100 $\mu \mathrm{M})$ significantly increased the acrosome reaction in porcine spermatozoa treated for $15 \min (P<0.05)$ (Figure $5 \mathrm{C}$ ), while no detectable effects were observed in mouse spermatozoa (Figure 5B). GEN also significantly increased the acrosome reaction in both mouse and porcine 

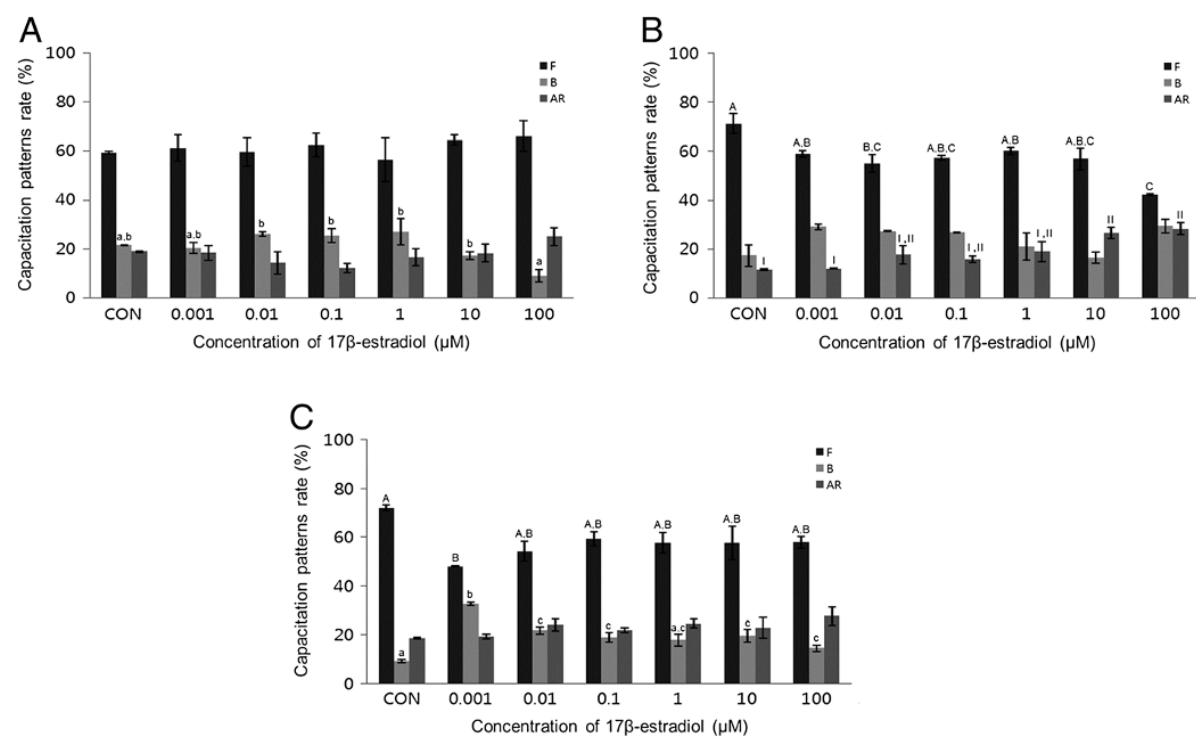

Figure 1 Effects of 15 min of incubation with $17 \beta$-estradiol (E2) on capacitation status. (A) Change of sperm capacitation status of bovine spermatozoa in the absence or presence of E2 $(0.001$ to $100 \mu \mathrm{M})$. (B) Change of sperm capacitation status of mouse spermatozoa in the absence or presence of E2 (0.001 to $100 \mu \mathrm{M})$. (C) Change of sperm capacitation status of porcine spermatozoa under in the absence or presence of E2 (0.001 to 100 MM). Capacitation status was distinguished F, B and AR pattern (Black Bar: F pattern, Grey Bar: B pattern, Dark-grey Bar: AR pattern). Data represent mean $\pm S E M, n=3$. $A, B, C$ Values with different superscripts were significantly different compared to control and the $F$ pattern group, by ANOVA $(P<0.05)$. ${ }^{a}, b, c$ Values with different superscripts were significantly different compared to control and the B pattern group, by ANOVA $(P<0.05)$. " "Values with different superscripts were significantly different compared to control and the AR pattern group, by ANOVA $(P<0.05)$.
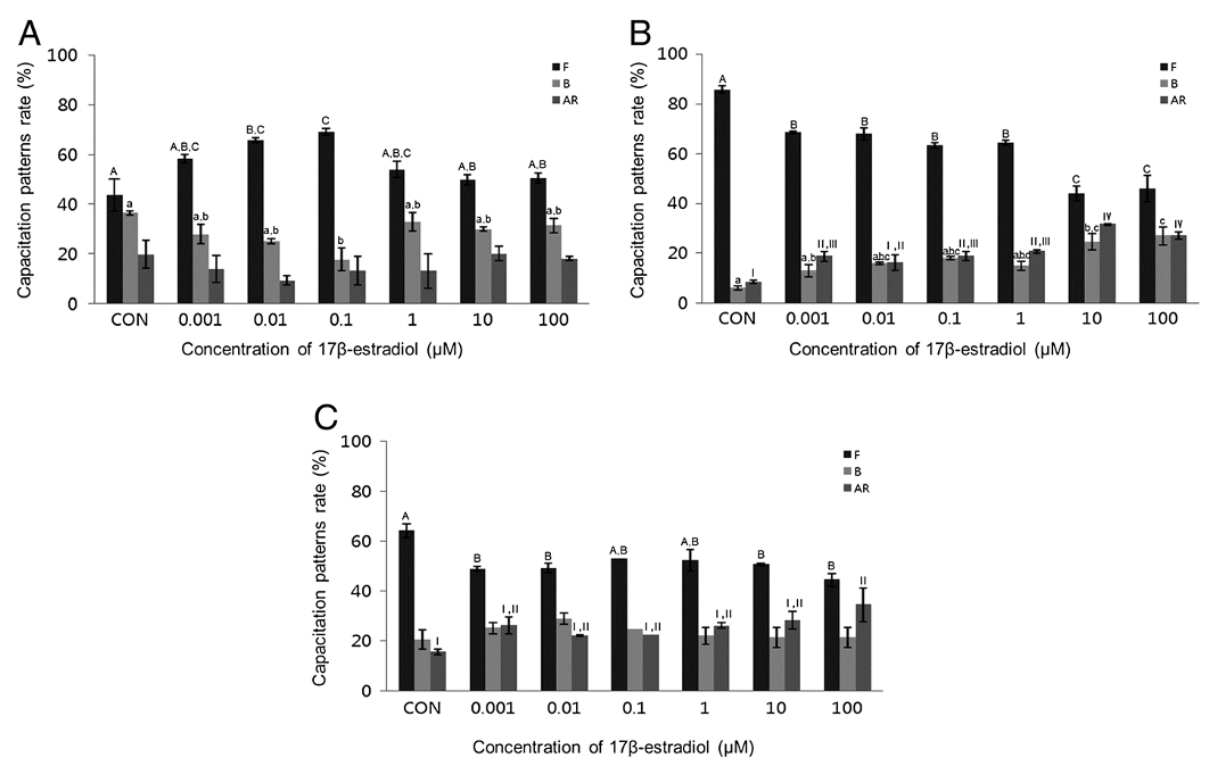

Figure 2 Effects of 30 min of incubation with $17 \beta$-estradiol (E2) on capacitation status. (A) Change of sperm capacitation status of bovine spermatozoa in the absence or presence of E2 $(0.001$ to $100 \mu \mathrm{M})$. (B) Change of sperm capacitation status of mouse spermatozoa in the absence or presence of E2 (0.001 to $100 \mu \mathrm{M})$. (C) Change of sperm capacitation status of porcine spermatozoa in the absence or presence of E2 (0.001 to 100 MM). Capacitation status was distinguished F, B and AR pattern (Black Bar: F pattern, Grey Bar: B pattern, Dark-grey Bar: AR pattern). Data represent mean $\pm S E M, n=3$. ${ }^{A}, B, C$ Values with different superscripts were significantly different compared to control and the $F$ pattern group, by ANOVA $(P<0.05) .{ }^{a}$, b, c Values with different superscripts were significantly different compared to control and the B pattern group, by ANOVA $(P<0.05)$. III III, IV Values with different superscripts were significantly different compared to control and the AR pattern group, by ANOVA $(P<0.05)$. 

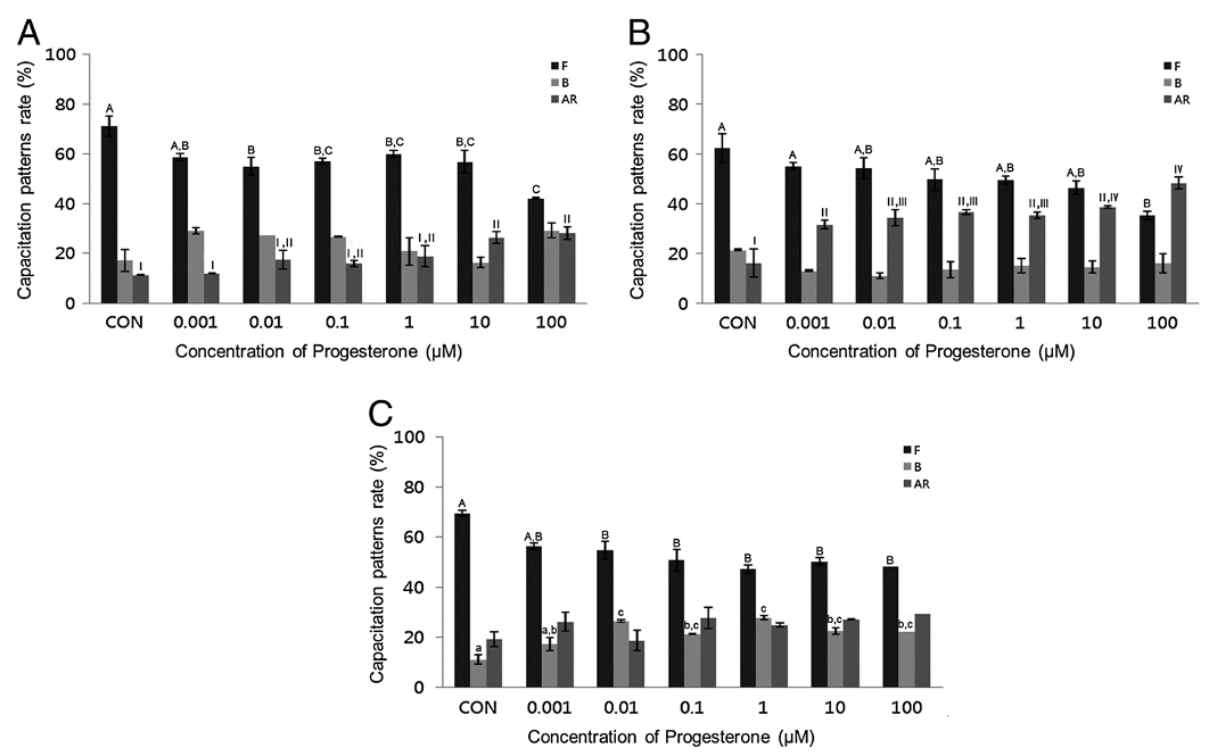

Figure 3 Effects of 15 min of incubation with progesterone (P4) on capacitation status. (A) Change of sperm capacitation status of bovine spermatozoa in the absence or presence of P4 (0.001 to $100 \mu \mathrm{M})$. (B) Change of sperm capacitation status of mouse spermatozoa in the absence or presence of P4 (0.001 to $100 \mu \mathrm{M})$. (C) Change of sperm capacitation status of porcine spermatozoa in the absence or presence of P4 (0.001 to 100 MM). Capacitation status was distinguished F, B and AR pattern (Black Bar: F pattern, Grey Bar: B pattern, Dark-grey Bar: AR pattern). Data represent mean $\pm S E M, n=3 .{ }^{A}, B, C$ Values with different superscripts were significantly different compared to control and the $F$ pattern group, by ANOVA $(P<0.05)$. ${ }^{a}$, b, c Values with different superscripts were significantly different compared to control and the B pattern group, by ANOVA $(P<0.05)$. III, III, IV Values with different superscripts were significantly different compared to control and the AR pattern group, by ANOVA $(P<0.05)$.
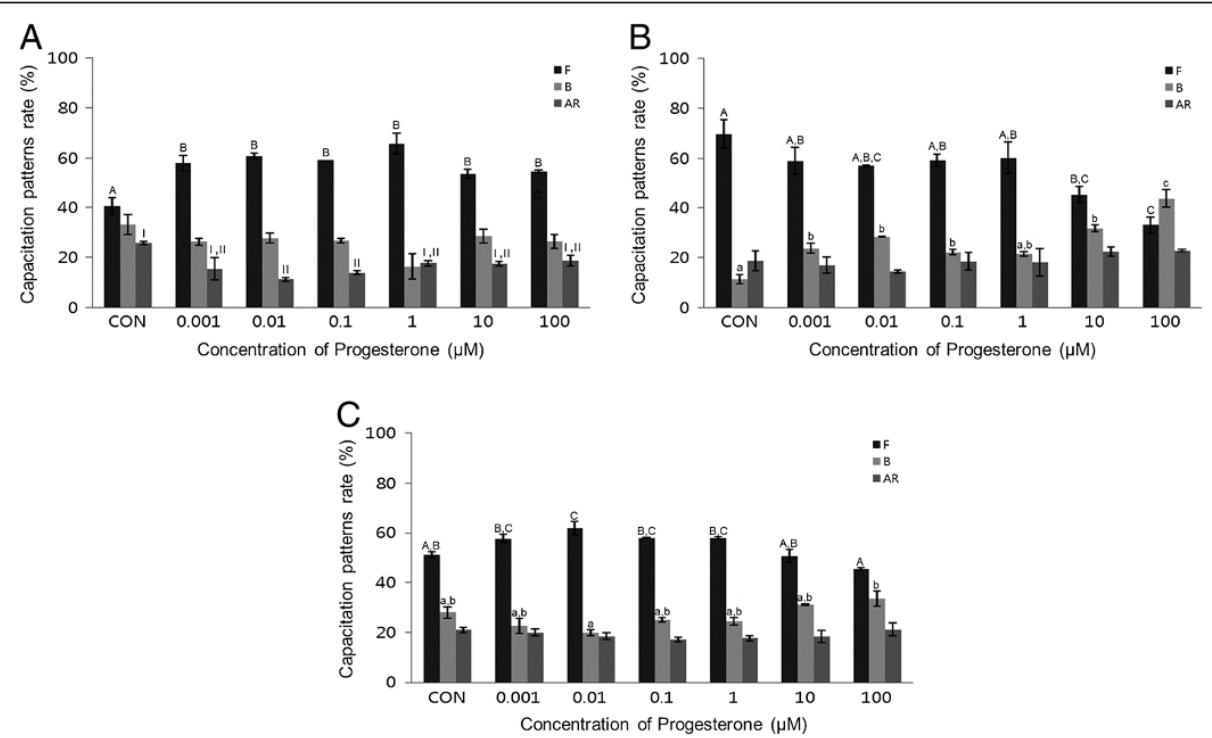

Figure 4 Effects of $\mathbf{3 0}$ min of incubation with progesterone (P4) on capacitation status. (A) Change of sperm capacitation status of bovine spermatozoa in the absence or presence of P4 (0.001 to $100 \mu \mathrm{M})$. (B) Change of sperm capacitation status of mouse spermatozoa in the absence or presence of P4 (0.001 to $100 \mu \mathrm{M})$. (C) Change of sperm capacitation status of porcine spermatozoa in the absence or presence of P4 (0.001 to 100 MM). Capacitation status was distinguished F, B and AR pattern (Black Bar: F pattern, Grey Bar: B pattern, Dark-grey Bar: AR pattern). Data represent mean $\pm S E M, n=3 .{ }^{A}, B, C$ Values with different superscripts were significantly different compared to control and the $F$ pattern group, by ANOVA $(P<0.05) .{ }^{\mathrm{a}, \mathrm{b}, \mathrm{c}}$ Values with different superscripts were significantly different compared to control and the $\mathrm{B}$ pattern group, by ANOVA $(P<0.05)$. " " Values with different superscripts were significantly different compared to control and the AR pattern group, by ANOVA $(P<0.05)$. 

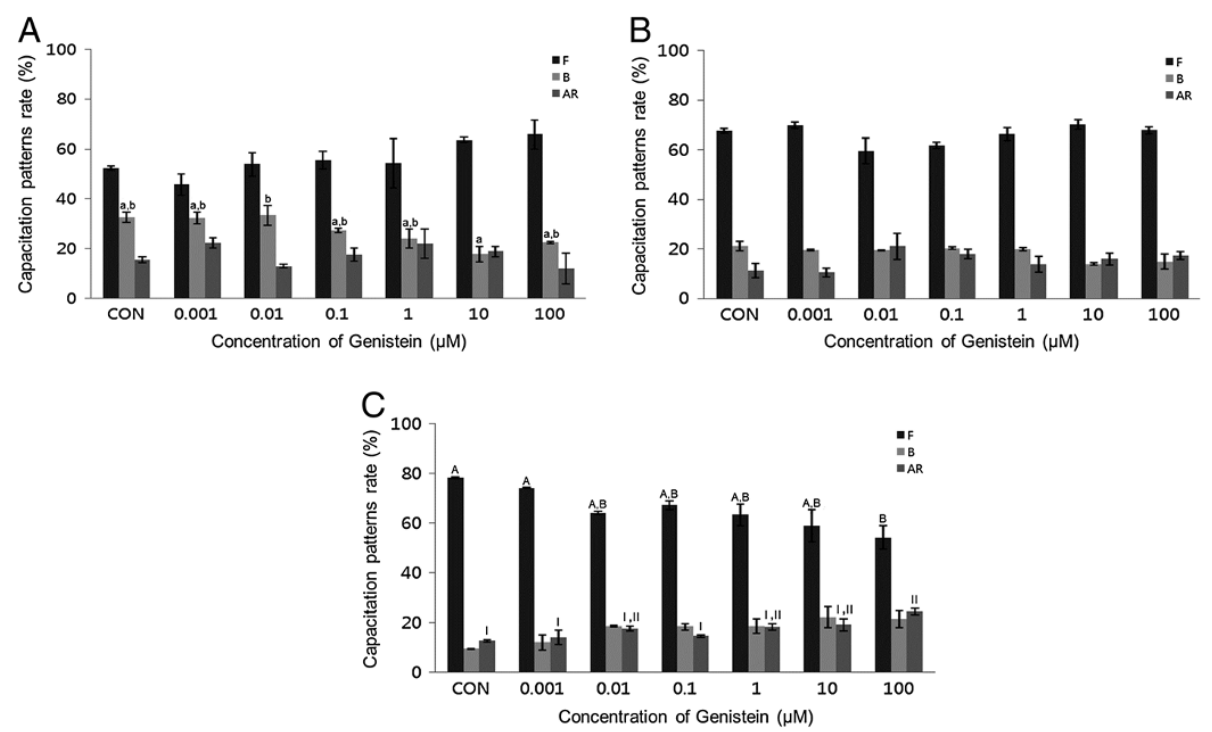

Figure 5 Effects of 15 min of incubation with genistein (GEN) on capacitation status. (A) Change of sperm capacitation status of bovine spermatozoa in the absence or presence of GEN $(0.001$ to $100 \mu \mathrm{M})$. (B) Change of sperm capacitation status of mouse spermatozoa in the absence or presence of GEN (0.001 to $100 \mu \mathrm{M})$. (C) Change of sperm capacitation status of porcine spermatozoa in the absence or presence of GEN (0.001 to $100 \mu \mathrm{M})$. Capacitation status was distinguished F, B and AR pattern (Black Bar: F pattern, Grey Bar: B pattern, Dark-grey Bar: AR pattern). Data represent mean $\pm S E M, n=3$. ${ }^{A}, B$ Values with different superscripts were significantly different compared to control and the $F$ pattern group, by ANOVA $(P<0.05)$. ${ }^{\mathrm{a}, \mathrm{b}}$ Values with different superscripts were significantly different compared to control and the B pattern group, by ANOVA $(P<0.05)$. " "Values with different superscripts were significantly different compared to control and the AR pattern group, by ANOVA $(P<0.05)$.

spermatozoa treated for $30 \mathrm{~min}(P<0.05)$ (Figure $6 \mathrm{~B}$ and $\mathrm{C}$ ). A concentration-dependent effect on the acrosome reaction was observed in both mouse and porcine spermatozoa. Capacitation was increased in bovine spermatozoa after $15 \mathrm{~min}$ incubation at a concentration of $0.001 \mu \mathrm{M}$. Upon treatment with $0.1 \mu \mathrm{M}$, this effect gradually decreased with increasing doses of GEN (Figure 5A). No detectable effect was observed after $30 \mathrm{~min}$ incubation.

Effects of OP on bovine, mouse, and porcine spermatozoa OP (0.001-100 $\mu \mathrm{M})$ significantly increased the acrosome reaction in mouse spermatozoa after $15 \min (P<0.05)$ (Figure 7B). This effect was dose-dependent manner. OP treatment also increased capacitation in porcine spermatozoa incubated for $15 \mathrm{~min}$ (Figure 7C). OP increased the acrosome reaction in bovine spermatozoa treated for $30 \mathrm{~min}$, however these differences were not significant except at $100 \mu \mathrm{M}$. No detectable effects were observed in mouse, porcine, or bovine spermatozoa (Figure 8).

\section{Discussion}

The present study addressed the question whether estrogens and endocrine disruptors interfere with bovine, mouse, and porcine spermatozoa function. Four chemicals, namely, E2, P4, GEN, and OP were evaluated at concentrations from 0.001 to $100 \mu \mathrm{M}$. Uncapacitated spermatozoa were treated for either 15 or $30 \mathrm{~min}$. We then assessed capacitation and the acrosome reaction using CTC analysis.

Interestingly, spermatozoa from mice, porcine, and bovine were responded to E2 in a different way. Mouse and porcine spermatozoa responded at different concentrations and times. Our results from mouse spermatozoa are in accordance with [10]. Unfortunately, we could not compare our findings from bovine and porcine with data from others because, to date, no such data has been collected by others. In various somatic cell systems, E2 has been reported to modulate $\mathrm{Ca}^{2+}$ fluxes, generate cyclic nucleotides, activate various kinases and modulate ion channels [14]. cAMP plays a role in spermatozoa physiology, many treatments that accelerate capacitation cause an increase in cAMP. cAMP plays a role in spermatozoa physiology many treatments that acceleration of capacitation cause by increasing in cAMP. Moreover, continuous stimulation of cAMP production appears to be associated with acrosome loss [10], these observations demonstrate the role of E2 in some of the physiological changes that take place in spermatozoa. Estrogens are classically thought to act by binding to estrogen receptors, ESR1 and ESR2 [15]. Notably, several studies have reported the presence of ERs on human [16,17] and rat [18] cell membranes. GEN and other estrogenic components are able to bind to both estrogen receptors, ESR1 and ESR2 [19-21]. These chemicals may exert their effects by binding to the same receptors. P4 significantly increased 

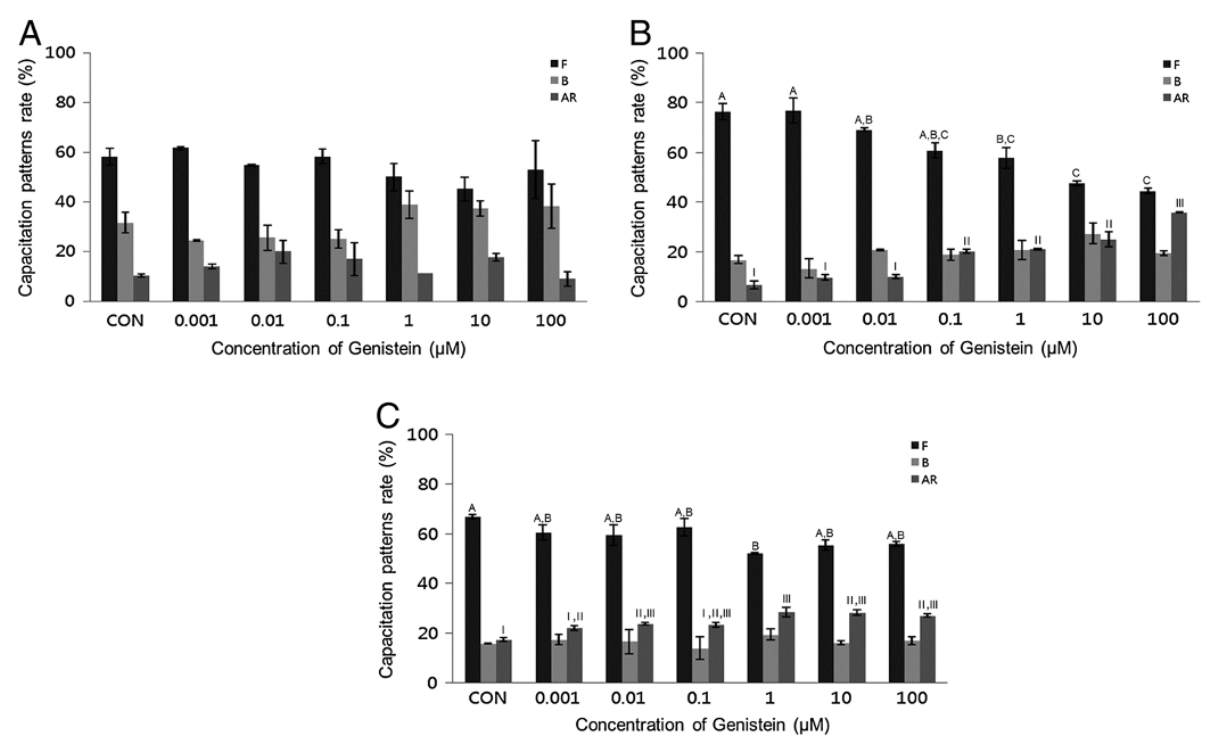

Figure 6 Effects of $30 \mathrm{~min}$ of incubation with genistein (GEN) on capacitation status. (A) Change of sperm capacitation status of bovine spermatozoa in the absence or presence of GEN $(0.001$ to $100 \mu \mathrm{M})$. (B) Change of sperm capacitation status of mouse spermatozoa in the absence or presence of GEN (0.001 to $100 \mu \mathrm{M})$. (C) Change of sperm capacitation status of porcine spermatozoa in the absence or presence of GEN (0.001 to $100 \mu \mathrm{M})$. Capacitation status was distinguished F, B and AR pattern (Black Bar: F pattern, Grey Bar: B pattern, Dark-grey Bar: AR pattern). Data represent mean $\pm S E M, n=3$. ${ }^{A}, B, C$ Values with different superscripts were significantly different compared to control and the $F$ pattern group, by ANOVA $(P<0.05)$. II, III Values with different superscripts were significantly different compared to control and the AR pattern group, by ANOVA $(P<0.05)$.
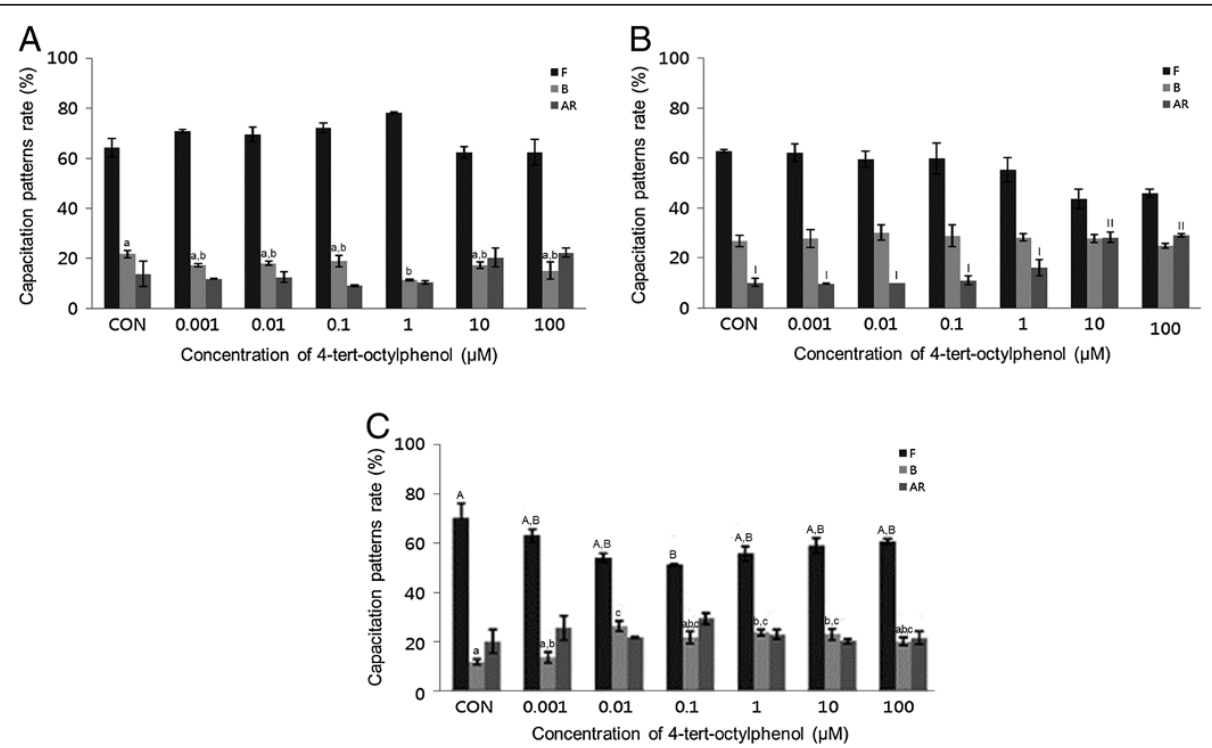

Figure 7 Effects of 15 min of incubation with 4-tert-octylphenol (OP) on capacitation status. (A) Change of sperm capacitation status of bovine spermatozoa in the absence or presence of OP $(0.001$ to $100 \mu \mathrm{M})$. (B) Change of sperm capacitation status of mouse spermatozoa in the absence or presence of OP $(0.001$ to $100 \mu \mathrm{M})$. (C) Change of sperm capacitation status of porcine spermatozoa in the absence or presence of OP (0.001 to $100 \mu M$ ). Capacitation status was distinguished F, B and AR pattern (Black Bar: F pattern, Grey Bar: B pattern, Dark-grey Bar: AR pattern). Data represent mean $\pm S E M, n=3$. ${ }^{A}, B$ Values with different superscripts were significantly different compared to control and the $F$ pattern group, by ANOVA $(P<0.05)$. ${ }^{a}$, b, c Values with different superscripts were significantly different compared to control and the B pattern group, by ANOVA $(P<0.05)$. " "Values with different superscripts were significantly different compared to control and the AR pattern group, by ANOVA $(P<0.05)$. 

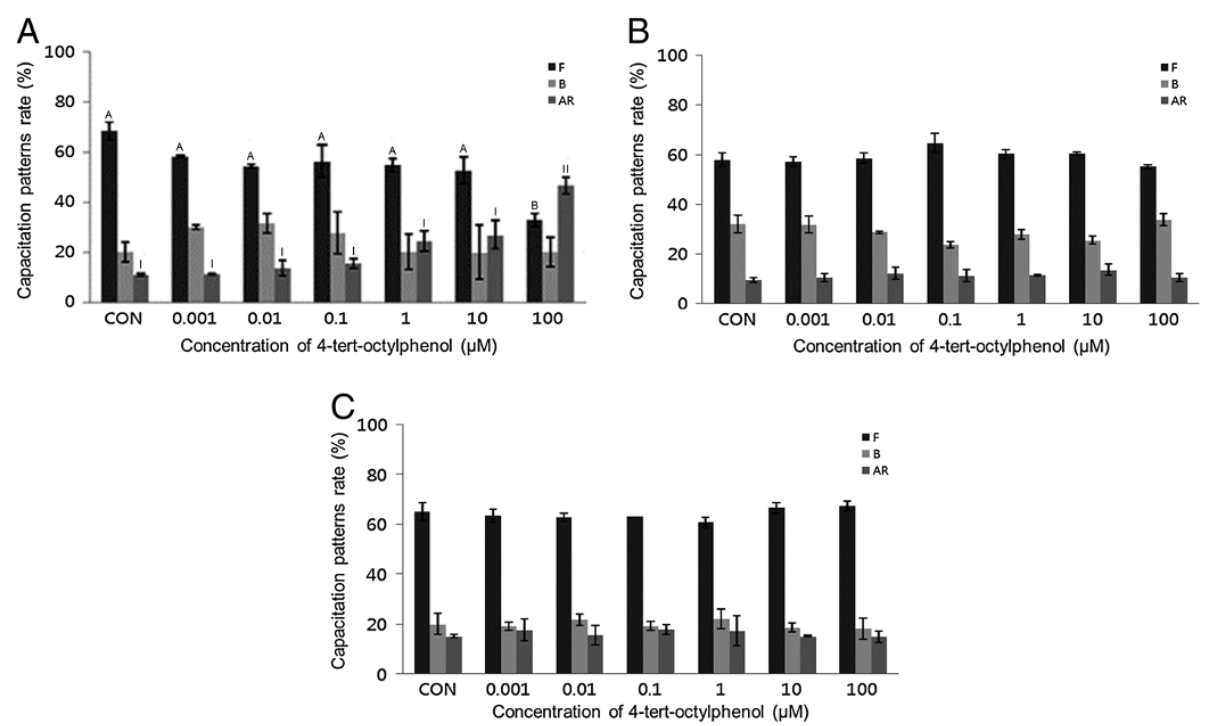

Figure 8 Effects of $15 \mathrm{~min}$ of incubation with 4-tert-octylphenol (OP) on capacitation status. (A) Change of sperm capacitation status of bovine spermatozoa in the absence or presence of OP $(0.001$ to $100 \mu \mathrm{M})$. (B) Change of sperm capacitation status of mouse spermatozoa in the absence or presence of OP $(0.001$ to $100 \mu \mathrm{M})$. (C) Change of sperm capacitation status of porcine spermatozoa in the absence or presence of OP (0.001 to $100 \mu \mathrm{M}$ ). Capacitation status was distinguished F, B and AR pattern (Black Bar: F pattern, Grey Bar: B pattern, Dark-grey Bar: AR pattern). Data represent mean $\pm S E M, n=3$. A, B Values with different superscripts were significantly different compared to control and the $F$ pattern group, by ANOVA $(P<0.05)$." " Values with different superscripts were significantly different compared to control and the AR pattern group, by ANOVA $(P<0.05)$.

the acrosome reaction in both bovine and mouse spermatozoa treated for $15 \mathrm{~min}(P<0.05)$ (Figure $3 \mathrm{~A}$ and $\mathrm{B}$ ), while significantly increased capacitation was observed in porcine spermatozoa $(P<0.05)$ (Figure $3 C)$. P4 significantly increased capacitation with dose-dependent manner in mouse spermatozoa treated for $30 \mathrm{~min}(\mathrm{P}<0.05)$ (Figure 4B), while no detectable effects were observed in either bovine or porcine spermatozoa. Spermatozoa from mice, porcine, and bovine were responded to $\mathrm{P} 4$ in the same way as they responded to E2. P4 seems to be involved in the physiological induction of the AR [22]. The changes induced by $\mathrm{P} 4$ require intercellular mechanisms dependent on protein kinase $\mathrm{C}$ and the $\mathrm{Ca}^{2+}$ channel. Unlike $\mathrm{Ca}^{2+}$ ionophores, $\mathrm{P} 4$ is a physiological inducer of the AR, because it does not bypass normal regulatory mechanisms [23]. In most species, P4 concentration within the reproductive tract fluids is still unknown $[24,25]$. In canine spermatozoa, the exposure of P4 binds with its receptors finally correlated with the maturation state of spermatozoa [26]. In stallions, the proportion of spermatozoa with exposed $\mathrm{P} 4$ receptors seems to correlate with the fertility of a given animal [27]. However, in bulls, $\mathrm{P} 4$ is not involved in the capacitation process, but rather initiates the AR, when spermatozoa are under capacitation conditions [28]. Others have reported that P4 significantly enhances sperm capacitation but does not significantly increase the AR of heparin-capacitated spermatozoa [29]. Therefore, our results indicate that P4 can induce the acrosome reaction in bovine and mouse spermatozoa, as well as stimulating capacitation in mouse and porcine spermatozoa. These findings are in contrast to other reports on bull spermatozoa that said $\mathrm{P} 4$ has a potential role in capacitation but not in AR. However, a significant time dependent increases of AR was reported in boar spermatozoa induced by P4 [30]. P4 also failed to accelerate capacitation or acrosome loss in human sperm suspensions pre-incubated for $1 \mathrm{~h}$ prior to a 30-min P4 treatment [10]. GEN significantly increased the acrosome reaction in both mouse and porcine spermatozoa treated for $30 \mathrm{~min}$ $(P<0.05)$ (Figure 6B and $C)$. A concentration-dependent effect on the acrosome reaction was observed in both mouse and porcine spermatozoa. GEN accelerated capacitation but this effect was not significant. Porcine spermatozoa responded to GEN in the same way as mouse spermatozoa did. In porcine spermatozoa, the acrosome reaction was increased at a concentration of $0.01 \mu \mathrm{M}$, but mouse spermatozoa required at least $0.1 \mu \mathrm{M}$ of GEN to elicit a significantly difference. Our results from mouse and porcine spermatozoa are in accordance with those of Fraser et al. [9], who reported that GEN accelerated capacitation and increased the acrosome reaction in human and mouse spermatozoa. While other investigators [19-21] have reported that GEN is able to bind to both estrogen receptors (ERs)-alpha and ER-beta, it has lower affinity than E2. Therefore, these observations were not in accordance with our data, which revealed similar effects for GEN and E2. However, other studies have reported that E2 and GEN both stimulate 
uncapacitated spermatozoa and that capacitation is associated with an increase in protein phosphorylation in humans [31,32]. Daidzein, like GEN, is an isoflavone associated with soy isoflavones and has been proven to have the same effect as GEN on uncapacitated mouse spermatozoa. Thus, soy isoflavones-containing products have at least two components with the potential to alter sperm function [33].

Finally, OP significantly increased the acrosome reaction in mouse spermatozoa, in a dose-dependent manner $(P<0.05)$ (Figures 7B and $8 \mathrm{~B})$, and significantly increased capacitation in porcine spermatozoa treated for $15 \mathrm{~min}$ $(P<0.05)$ (Figure 7C). Bovine spermatozoa showed significant increases in the AR following 30-minute incubation at the highest concentration of OP studied $(P<0.05)$ (Figure 8A). However, no detectable effects were observed in mouse or porcine spermatozoa following incubation for $30 \mathrm{~min}$. While OP has been reported to be estrogenic in fish and mammalian cells and mimics the effect of E2 by binding to the estrogen receptor [34], our data revealed that OP had low estrogenic activity in all spermatozoa tested. This was in accordance with Aydogan et al. [34], who reported that the estrogenic activity of OP was approximately $10^{-3}$ to $10^{-7} \mathrm{M}$ relative to E2. The low efficiency of OP may result from the lower affinity with which it binds to ERs [19-21]. Luconi et al. [2] also reported that octyphenol polyethoxylate had no detectable effect in humans.

\section{Conclusions}

All chemicals studied effectively altered capacitation and the acrosome reaction in bovine, mouse, and porcine spermatozoa. However, when spermatozoa were incubated for 15 or $30 \mathrm{~min}$ in all chemicals studied, capacitation status and acrosome reaction were significantly different in the responsiveness of bovine, mouse and porcine spermatozoa to E2, P4, GEN, and OP were significantly different. Porcine spermatozoa were more responsive than the other spermatozoa. Therefore, we suggest that porcine spermatozoa can be used as a suitable tool for in vitro screening of potential endocrine disruptors.

\section{Competing interests}

The authors declare that they have no competing interests.

\section{Authors' contributions \\ Conceived and designed the experiments: DYR, YJK, MGP. Performed the experiments: DYR, YJK, JSL, MSR. Analyzed the data: DYR, YJK, MSR, WSK, SJY, MGP. Contributed reagents/materials/analysis tools: DYR, YJK, MGP. Wrote the paper: DYR, YJK, MGP. All authors read and approved the final manuscript.}

\section{Acknowledgement}

This study was financially supported by the Cooperative Research Program for Agriculture Science and Technology Development (Project no. PJ008415), Rural Development Administration, Republic of Korea.
Received: 22 August 2014 Accepted: 17 October 2014

Published: 27 November 2014

\section{References}

1. Stormshak F, Bishop CV: Estrogen and progesterone signaling: genomic and nongenomic actions in domestic ruminants. J Anim Sci 2008, 86:299-315.

2. Luconi M, Muratori M, Forti G, Baldi E: Identification and characterization of a novel functional estrogen receptor on human sperm membrane that interferes with progesterone effects. J Clin Endocrinol Metab 1999, 84:1670-1678.

3. Couse JF, Korach KS: Estrogen receptor null mice: what have we learned and where will they lead us? Endocr Rev 1999, 20:358-417.

4. Sharpe RM: Hormones and testis development and the possible adverse effects of environmental chemicals. Toxicol Lett 2001, 120:221-232.

5. Skakkebaek NE, Rajpert-De Meyts E, Main KM: Testicular dysgenesis syndrome: an increasingly common developmental disorder with environmental aspects. Hum Reprod 2001, 16:972-978.

6. Sohoni P, Sumpter JP: Several environmental oestrogens are also anti-androgens. J Endocrinol 1998, 158:327-339.

7. Akingbemi BT, Hardy MP: Oestrogenic and antiandrogenic chemicals in the environment: effects on male reproductive health. Ann Med 2001, 33:391-403.

8. Luconi M, Bonaccorsi L, Forti G, Baldi E: Effects of estrogenic compounds on human spermatozoa: evidence for interaction with a nongenomic receptor for estrogen on human sperm membrane. Mol Cell Endocrinol 2001, 178:39-45.

9. Fraser LR, Beyret E, Milligan SR, Adeoya-Osiguwa SA: Effects of estrogenic xenobiotics on human and mouse spermatozoa. Hum Reprod 2006, 21:1184-1193

10. Adeoya-Osiguwa SA, Markoulaki S, Pocock V, Milligan SR, Fraser LR: 17beta-Estradiol and environmental estrogens significantly affect mammalian sperm function. Hum Reprod 2003, 18:100-107.

11. Park YJ, Mohamed e-SA, Kwon WS, You YA, Ryu BY, Pang MG: Xenoestrogenic chemicals effectively alter sperm functional behavior in mice. Reprod Toxicol 2011, 32(4):418-424.

12. Perez $L$, Valcarcel A, de Las Heras MA, Mouse DF, Baldassarre H: In vitro capacitation and induction of acrosomal exocytosis in ram spermatozoa as assessed by the chlortracyline assay. Theriogenology 1996, 45:1037-1046.

13. Maxwell WM, Johnson LA: Physiology of spermatozoa at high dilution rates: the influence of seminal plasma. Therigenology 1999, 52:1353-1362.

14. Kelly MJ, Levin ER: Rapid action of plasma membrane estrogen receptor. Trends Endocrinol Metab 2001, 12:152-156.

15. Sirianni R, Chimento A, Ruggiero C, De Luca A, Lappano R, Andò S, Maggiolini M, Pezzi V: The novel estrogen receptor, G protein-coupled receptor 30 , mediates the proliferative effects induced by 17 betaestradiol on mouse spermatogonial GC-1 cell line. Endocrinology 2008, 149:5043-5051.

16. Misao R, Niwa K, Morishita S, Fujimoto J, Nakanishi Y, Tamaya T: Immunohistochemical detection of estrogen and progesterone receptors in spermatozoa of infertile men. Int J Fertil 1997, 42:421-425.

17. Durkee TJ, Mueller M, Zinaman M: Identification of estrogen receptor protein and messenger ribonucleic acid in human spermatozoa. Am J Obstet Gynecol 1998, 178:1288-1297.

18. Saberwal GS, Sharma MK, Balasinor N, Choudhary J, Juneja HS: Estrogen receptor, calcium mobilization and rat sperm motility. Mol Cell Biochem 2002, 237:11-20

19. Kuiper GG, Carlsson B, Grandien K, Enmark E, Haggblad J, Nilsson S, Gustafsson JA: Comparison of the ligand binding specificity and transcript tissue distribution of estrogen receptors alpha and beta. Endocrinology 1998, 138:863-870.

20. Milligan SR, Kalita JC, Heyerick A, Rong H, De Cooman L, De Keukeleire D: Identification of a potent phytoestrogen in hops (Humulus lupulus L.) and beer. J Clin Endocrinol Metab 1999, 83:2249-2252.

21. Milligan SR, Kalita JC, Pocock V, Van de Kauter V, Stevens JF, Deinzer ML, Rong H, De Keukeleire D: The endocrine activities of 8-prenylnaringenin and related hop (Humulus lupulus I.) flavonoids. J Clin Endocrinol Metab 2000, 85:4912-4915.

22. Neild DN, Gadella BM, Aguero A, Stout TAE, Colenbrander B: Capacitation, acrosome finction and chromatin structure in stallion sperm. Anim Reprod Sci 2005, 89:47-56. 
23. Witte TS, Schäfer-Somi S: Involvement of cholesterol, calcium and progesterone in the induction of capacitation and acrosome reaction of mammalian spermatozoa. Anim Reprod Sci 2007, 102:181-193.

24. Saaranen MJ, Calvo L, Dennison L, Banks S, Bustillon AD, Dorfmann M, Goldstein M, Thorsell L, Schulmann JD, Sherins RJ: Acrosome reaction inducing activity in follicular fluids correlates with progesterone concentration but not with oocyte maturity or fertilizability. Hum Reprod 1993, 8:1448-1454

25. Libersky EA, Boatman DE: Progesterone concentrations in serum, follicular fluid, and oviductal fluid of the golden hamster during periovulatory period. Biol Reprod 1995, 53:477-482.

26. Sirivaidyapong S, Bevers MM, Gadella BM, Colenbrander B: Induction of the acrosome reaction in dog sperm cells is dependent on epididymal maturation: the generation of a functional progesterone receptor is involved. Mol Reprod Dev 2001, 58:451-459.

27. Rathi R, Colenbrander B, Stout TAE, Bevers MM, Gadella BM: Progesterone induces acrosome reaction in stallion spermatozoa via a protein tyrosine kinase dependent pathway. Mol Reprod Dev 2003, 64:120-128.

28. Therien I, Manjunath P: Effect of progesterone on bovine sperm capacitation and acrosome reaction. Biol Reprod 2003, 69:1408-1415.

29. Lucoseviciute $K$, Zilinskas $H$, Januskauskas A: Effect of exogenous progesterone on post-thaw capacitation and acrosome reaction of bovine spermatozoa. Reprod Dom Anim 2004, 39:154-161.

30. Wu JT, Chiang KC, Cheng FP: Expression of progesterone receptor(s) during capacitation and incidence of acrosome reaction induced by progesterone and zona proteins in boar spermatozoa. Anim Reprod SC 2006, 93:24-45.

31. Visconti PE, Moore GD, Bailey JL, Leclerc P, Connors SA, Pan D, Olds-Clarke P, Kopf GS: Capacitation of mouse spermatozoa. II: protein tyrosine phosphorylation and capacitation are regulated by a CAMP-dependent pathway. Development 1995, 121:1139-1150

32. Adeoya-Osiguwa SA, Fraser LR: Fertilization promoting peptide and adenosine, acting as first messengers, regulate cAMP production and consequent protein tyrosine phosphorylation in a capacitation-dependent manner. Mol Reprod Dev 2000, 57:384-392.

33. Pukazhenthi BS, Wildt DE, Ottinger MA, Howard J: Inhibition of domestic cat spermatozoa acrosome reaction and zona pellucida penetration by tyrosine kinase inhibitors. Mol Reprod Dev 1998, 49:48-57.

34. Aydogan M, Barlas N: Effect of maternal 4-tert-octylphenol exposure on the reproductive tract of male rats and adulthood. Reprod Toxicol 2006, 22:455-460.

doi:10.1186/2055-0391-56-26

Cite this article as: Ryu et al:: Capacitation and acrosome reaction differences of bovine, mouse and porcine spermatozoa in responsiveness to estrogenic compounds. Journal of Animal Science and Technology 2014 56:26.

\section{Submit your next manuscript to BioMed Central and take full advantage of:}

- Convenient online submission

- Thorough peer review

- No space constraints or color figure charges

- Immediate publication on acceptance

- Inclusion in PubMed, CAS, Scopus and Google Scholar

- Research which is freely available for redistribution 